\title{
Wavelet Transform Based Higher Order Statistical Analysis of Wind and Wave Time Histories
}

\author{
Gulamhusenwala Habib Huseni $^{1}$ - Ramakrishnan Balaji ${ }^{2}$
}

Received: 29 October 2014/ Accepted: 18 May 2016/Published online: 21 June 2016

(C) The Institution of Engineers (India) 2016

\begin{abstract}
Wind, blowing on the surface of the ocean, imparts the energy to generate the waves. Understanding the wind-wave interactions is essential for an oceanographer. This study involves higher order spectral analyses of wind speeds and significant wave height time histories, extracted from European Centre for Medium-Range Weather Forecast database at an offshore location off Mumbai coast, through continuous wavelet transform. The time histories were divided by the seasons; pre-monsoon, monsoon, post-monsoon and winter and the analysis were carried out to the individual data sets, to assess the effect of various seasons on the wind-wave interactions. The analysis revealed that the frequency coupling of wind speeds and wave heights of various seasons. The details of data, analysing technique and results are presented in this paper.
\end{abstract}

Keywords Wavelet transformation - Bispectrum .

Waves - Monsoon

\section{Introduction}

Surface waves on the ocean waters, generated by winds, are of great interest to engineers and scientists. The linear and non-linear interactions of wind speed and wave characteristics reveal the influences of the wind on the wave generations and growth. In general the wind and wave time histories are analysed through

Ramakrishnan Balaji

rbalaji@iitb.ac.in

University of Illinois, Urbana 61801, Illinois, USA

2 Indian Institute of Technology Bombay, Mumbai 400076, Maharashtra, India various transformation techniques to estimate their spectral characteristics. In recent times, the application of wavelet based transformation of various ocean parameters has found to be successful [1-5]. The wavelet based bicoherences were estimated to analyse the non-linear wave-wave interactions of a measured wave elevation time history [6]. Further, the phase coupling and nonlinear interactions of the wind speeds and wave elevations over certain frequencies were identified using the wavelet based bicoherences [7]. In another study, the linear and nonlinear wind-wave interactions of a simultaneously measured wind and wave datasets were analysed using wavelet linear coherence and wavelet bicoherence respectively [8]. The study also demonstrated the advantages of dividing the wind-wave time histories into discrete segments for better exploration of linear and nonlinear wind-wave phase couplings. Using simultaneously measured wind velocities and wave elevations, the linear and nonlinear interactions between the wind fluctuations and the wave field through Fourier based bispectral analysis were studied [9].

In this study, the wind speeds and wave heights time histories, extracted off Mumbai coastline for a particular year were subjected to wavelet based linear coherence analysis to understand the interactions among the metocean parameters. It is well know that the meteorological parameters of the four major seasons; pre-monsoon (March to May), monsoon (June to September), post-monsoon (October to December) and winter (January to February), are dynamically varying along the Indian coast. Hence, the time histories were segmented into season-wise and analysed to understand the effect of seasons on the interactions. The data, methodology of analysis and results are presented in this paper. 


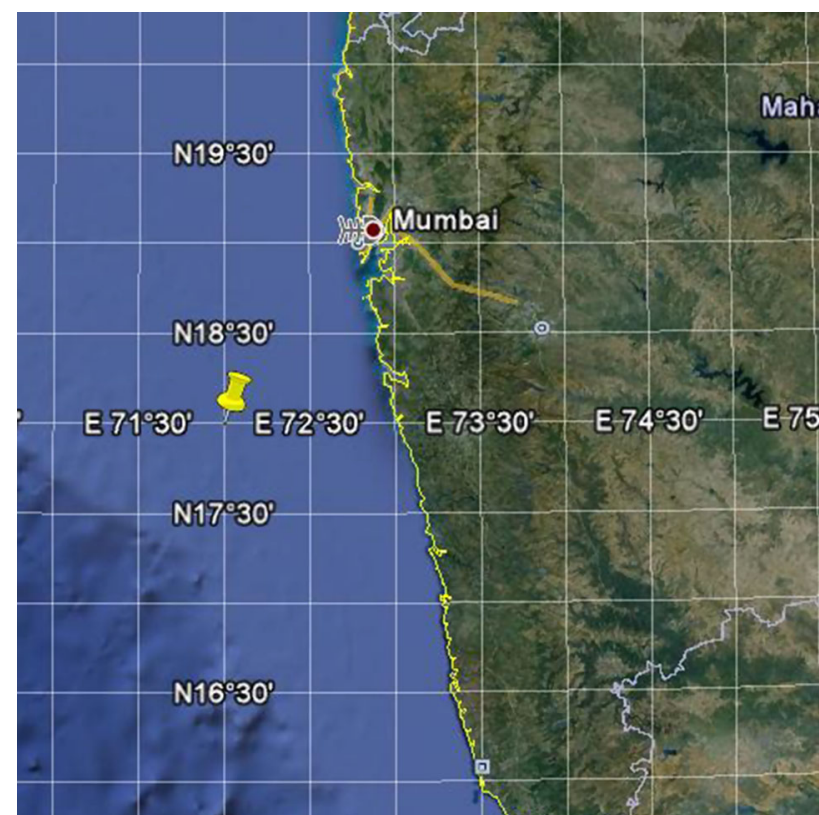

Fig. 1 Location of data extraction

\section{Materials and Methods}

In order to assess the interaction, season-wise time histories of wind speed and significant wave heights were extracted from European Centre for Medium-Range Weather Forecast (ECMWF) for the year 2012. ECMWF uses combined numerical weather prediction tools and constant input of meteorological data, collected by various systems such as satellites, aircraft, ships and weather balloons. The wind and wave data obtained from ECMWF are available at a spatial resolution of $1.5^{\circ}$ in longitude and latitude. ECMWF estimate forecasted wind and wave data sets, for medium range (15 days), monthly and seasonal. The accuracy of the ECMWF data has been verified with the observational buoy data-sets, for Indian coastline and has been found in good agreement [10]. The datasets from ECMWF are available at a regular time interval and were extracted at an offshore location $\left(7^{\circ} \mathrm{N} 57^{\circ} \mathrm{E} 25^{\circ} \mathrm{N} 79^{\circ} \mathrm{E}\right)$ off Mumbai coastline, as indicated in Fig. 1. The extracted wind speeds and significant wave height, shown in Figs. 2 and 3 respectively, clearly indicate the large seasonal
Fig. 2 Typical season-wise wind speeds
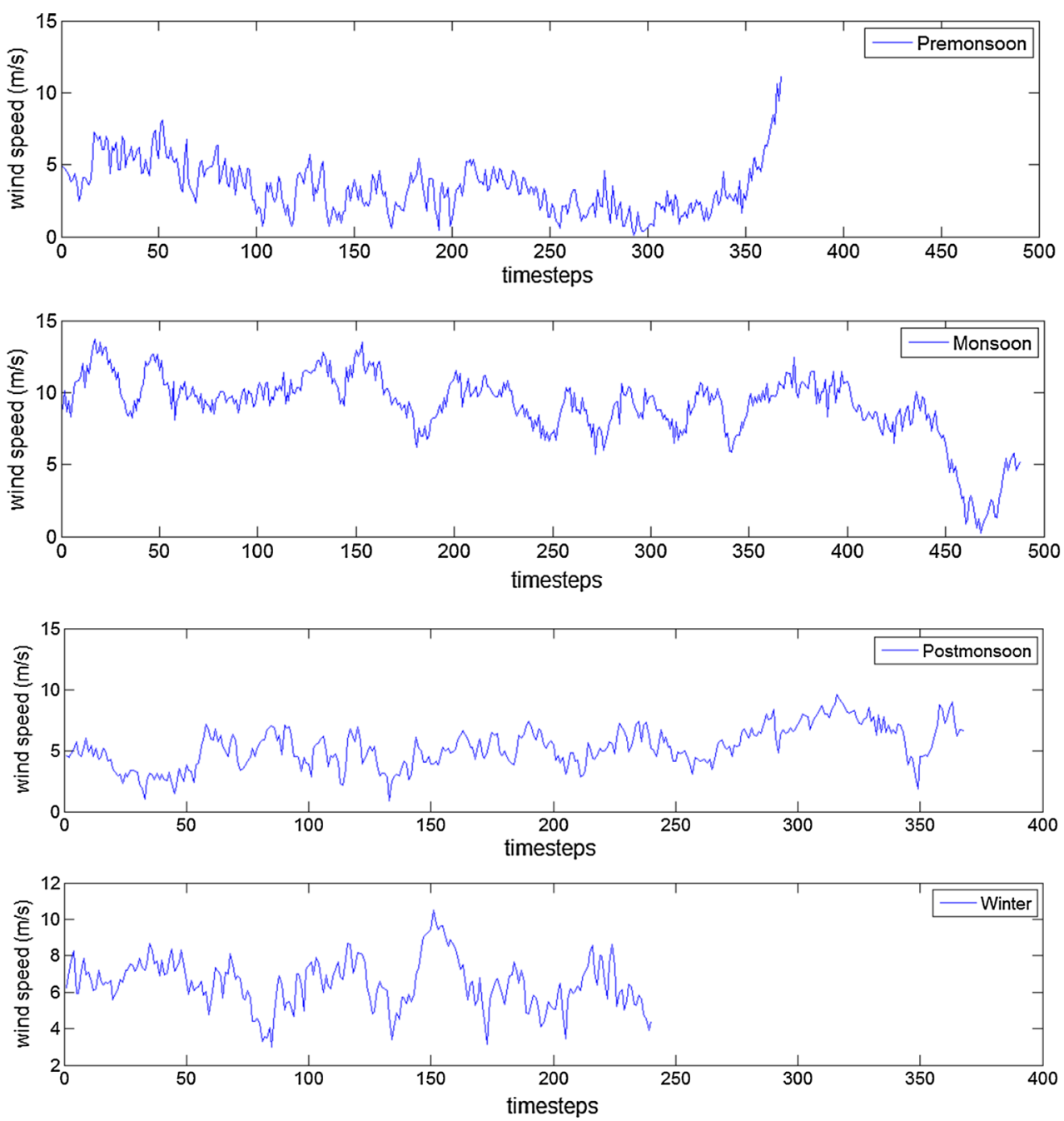
Fig. 3 Typical season-wise significant wave heights
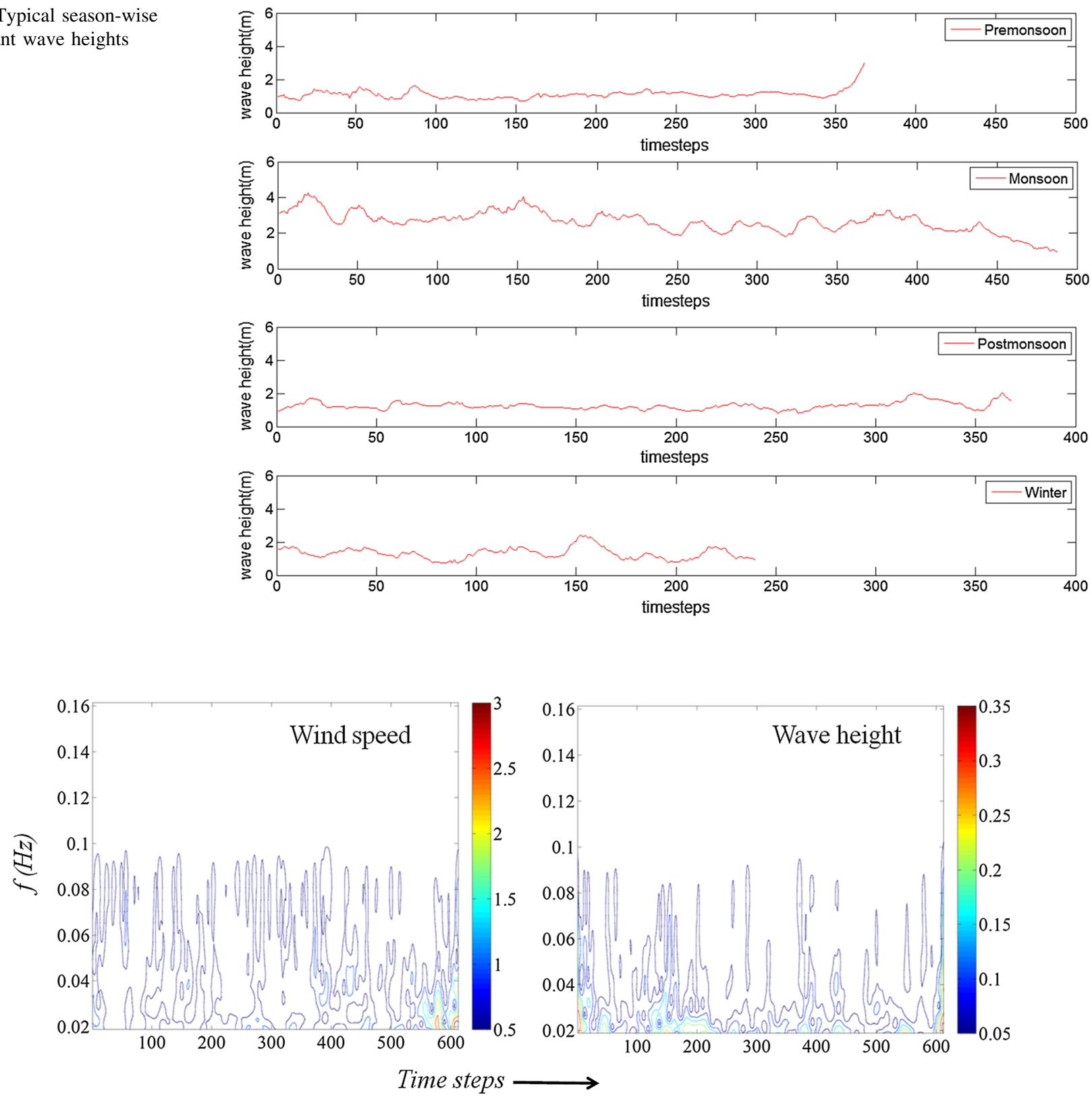

Fig. 4 Typical continuous wavelet transforms of time series of monsoon season

variations in their characteristics. The time histories were then subjected to continuous wavelet transform (CWT), in which Morlet wavelet is adopted as the mother wavelet. The CWT of a one-dimensional signal $x(t)$ is given as,

$W_{x}(a, \tau)=\frac{1}{\sqrt{|a|}} \int_{-\infty}^{\infty} x(t) \cdot \psi^{*}\left(\frac{t-\tau}{a}\right) \mathrm{d} t$

in which, $\psi^{*}$ is scaled $(a)$ and translated $(\tau)$ version of mother wavelet (basis function) $\psi$. The usage of Morlet wavelet as basis function for the wavelet transformation of ocean wave signals is quite common and hence adopted in this present study also. The Morlet wavelet function is a
Gaussian modulated complex-valued plane wave, which is defined as,

$\psi(t)=\pi^{-(1 / 4)} e^{i \omega_{0} t} e^{-\left(t^{2} / 2\right)}$

where $\varpi_{0}$ is the non-dimensional frequency, suggested as 6.0 [11]. Further, the wavelet power spectra were estimated as;

$S_{x x}=\int_{T} W_{x}^{*}(a, \tau) W_{x}(a, \tau) \mathrm{d} \tau$

in which, $W_{x}^{*}(a, \tau)$ is complex conjugate of wavelet transform. The cross spectrum of the wind and wave time 

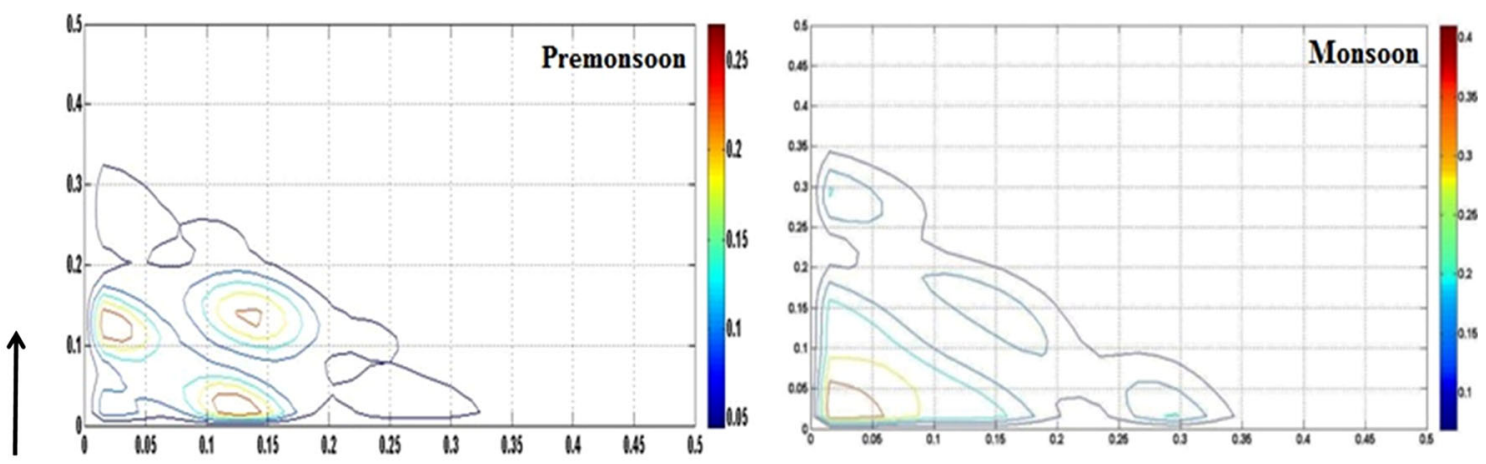

ज्ञ
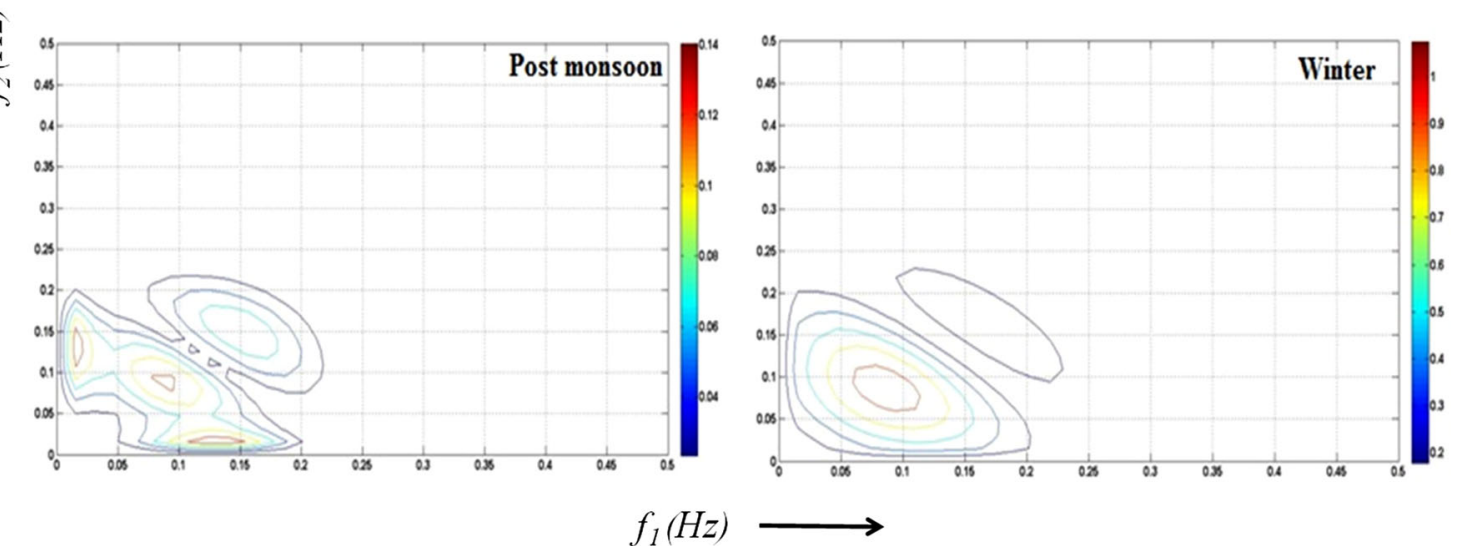

Fig. 5 Bispectra of wind speeds
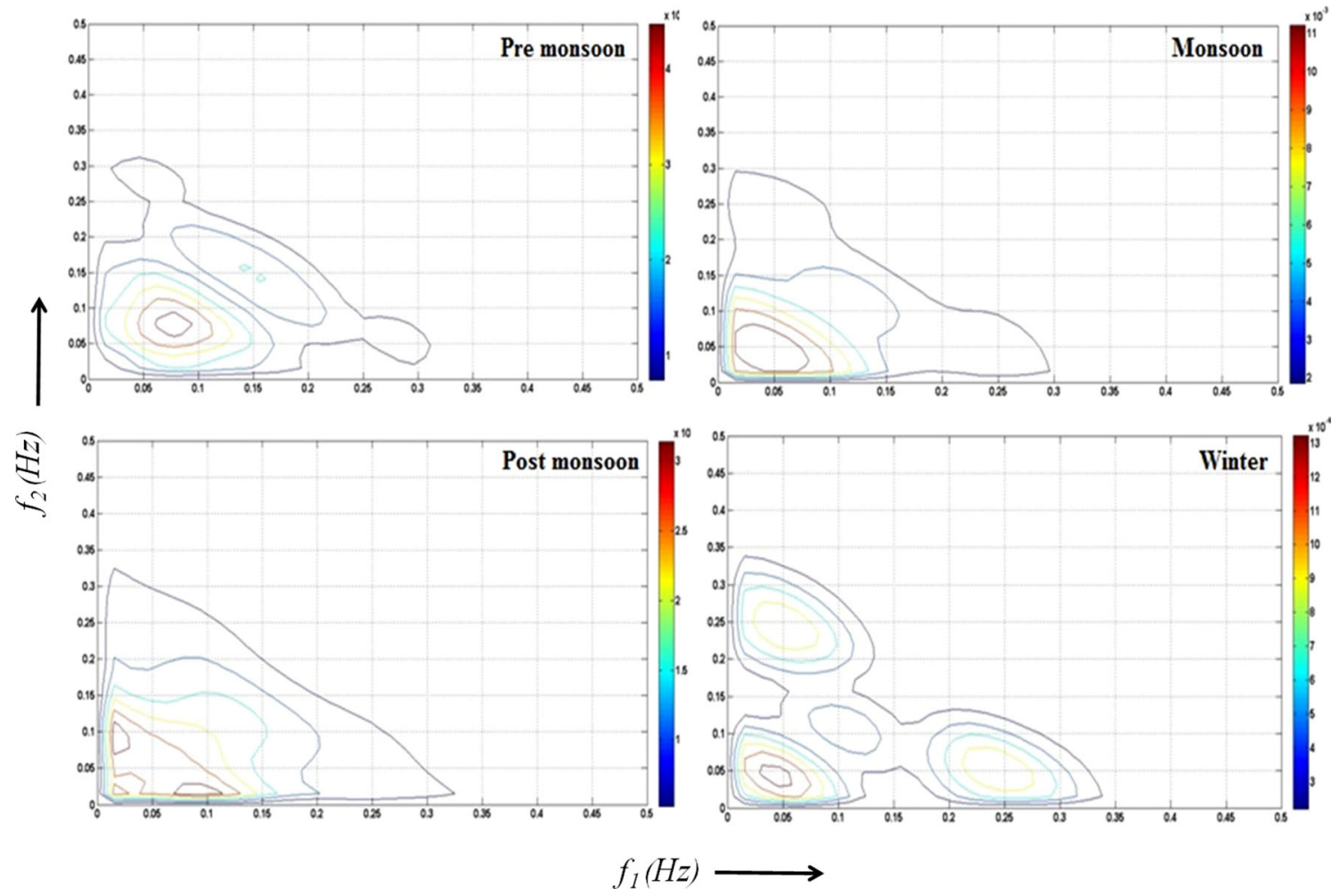

Fig. 6 Bispectra of wave heights 
histories, to determines the relation between them in terms of their frequency components, were then given by;

$S_{x y}=\int_{T} W_{x}^{*}(a, \tau) W_{y}(a, \tau) \mathrm{d} \tau$

where, $W_{x}^{*}(a, \tau)$ and $W_{y}(a, \tau)$ are complex conjugate of wave heights and wavelet transform of the wind speed time series respectively. The wavelet transform based linear coherence is then given by;

$C_{x y}(a)=\frac{\left|S_{x y}(a)\right|^{2}}{S_{x x}(a) S_{y y}(a)}$

Based on the estimated wavelet transform of a time history, the bispectrumcan be obtained as;

$B_{x x x}(a 1, a 2)=\int_{T} W_{x}(a 1, \tau) W_{x}(a 2, \tau) W_{x}^{*}(a 1+a 2, \tau) \mathrm{d} \tau$

In the above equation, $a 1$ and $a 2$ are scales that follow frequency sum rule. The analysis of the datasets and the results are discussed in the following section.

\section{Results and Discussion}

Initially, the wind speed and significant wave height time histories, extracted from ECMWF at a particular location offshore of Mumbai coastline, were systematically subjected to CWT, as defined in Eq. (1), to obtain the wavelet spectra. The CWT, basically, gives contours of wavelet power spectra in time and frequency domain, as typically shown for wind and wave data for the monsoon season, in Fig. 4. It is observed from the wavelet contours that the range of frequencies of wind and wave are varied from 0.02 to $0.1 \mathrm{~Hz}$.

The data sets were further subjected to cross-spectral analysis (Eq. 4) to obtain the cross-spectra of wind speeds and wave heights. As the objective of the study is to identify the frequency coupling in the wind speeds and wave heights, the data sets were then subjected to wavelet based bispectral analysis (Eq. 6). The contours of bispectra of all the seasonal datasets are shown in Figs. 5 and 6 for wind and waves respectively. It is observed from the autobispectra of wind speeds that the two coupling pattern clearly visible in pre-monsoon and post-monsoon periods are reduced to a single coupling for monsoon and winter
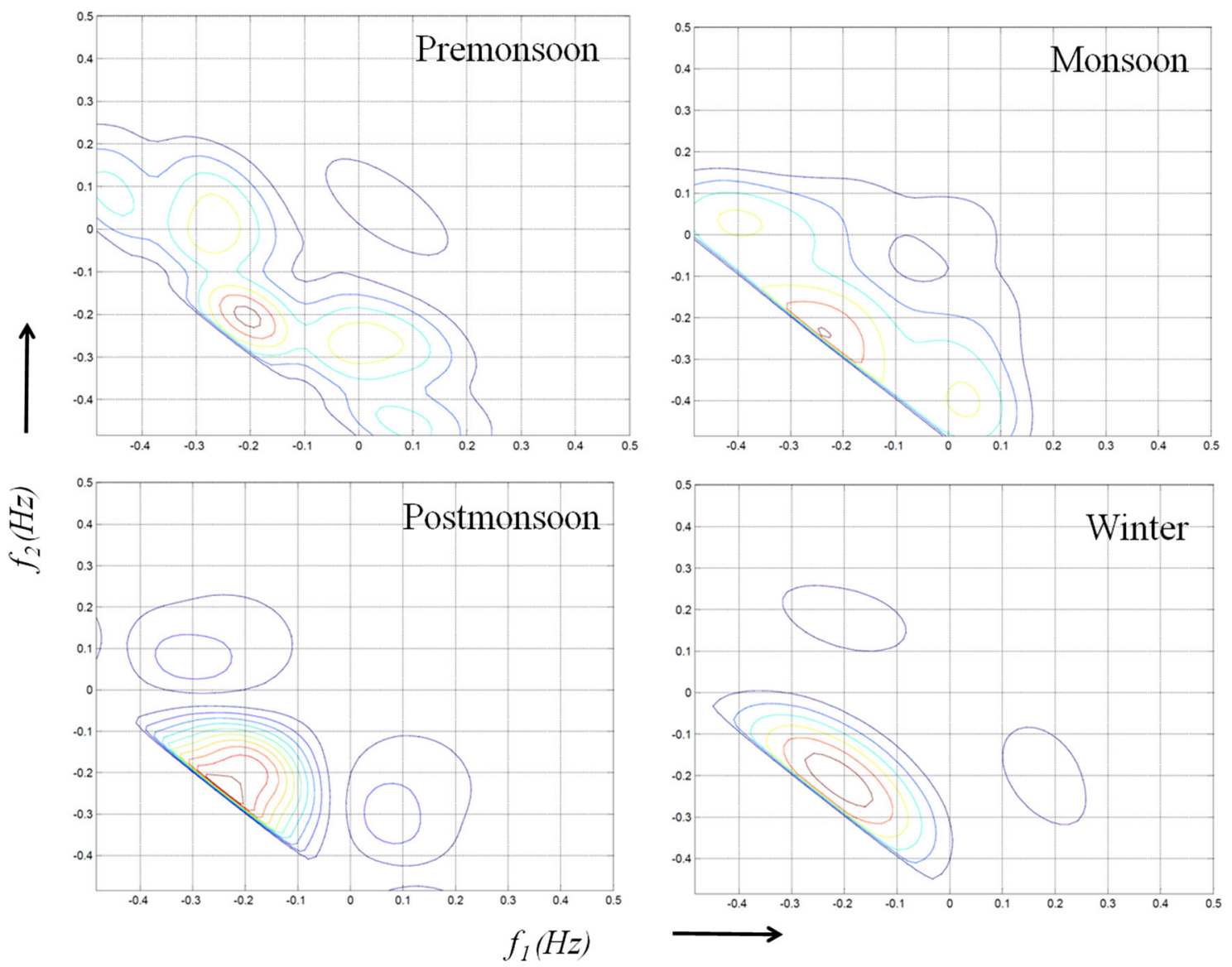

Fig. 7 Cross bispectraof wind-wave 
seasons. The coupling frequencies of the pre-monsoon, monsoon, post-monsoon and winter were observed in the ranges of $(0.025,0.125),(0.14,0.14),(0.025,0.025)$, $(0.125,0.025),(0.08,0.08)$ and $(0.075,0.075)$, respectively for the wind speeds. For the wave heights, the frequency couplings are observed as $(0.075,0.075),(0.04,0.04),(0.1$, $0.025),(0.025,0.025)$ and $(0.05,0.05),(0.25,0.05)$ respectively for four seasons.

In order to assess the effect of wind speeds on the wave heights and subsequent frequency couplings, the data sets were then subjected to wavelet based cross bispectral analysis. The cross bispectra of wind and wave datasets obtained for all the four seasons are shown in Fig. 7. It is clear from Fig. 7 that the spectral energies are observed to be high at different frequencies, indicating the active interactions between wind and wave. The maximum spectral peak is found to be at $(0.2,0.2)$ for all the seasons.

\section{Conclusion}

Wavelet based spectral analysis of wind speeds and wave heights, extracted from ECMWF, has been carried out to understand the frequency interactions. The bispectral and cross bispectral analysis of the winds and waves revealed the various frequency combinations that interact with in the time histories of wind speeds and wave heights. The results of the study primarily explain the interesting features of wind energy transfer and wave growth at various frequency ranges at a particular place of interest. In addition, the application of wavelet transformation based higher order statistics of met-ocean parameters is demonstrated in this paper.
Acknowledgments This research work was supported through Summer Research Fellowship Programme funded by Indian Academy of Science for the year 2012.

\section{References}

1. S.R. Massel, Wavelet analysis for processing of ocean surface wave records. Ocean Eng. 28(8), 957-987 (2001)

2. R. Balaji, S. Sannasiraj, V. Sundar, Laboratory simulation and analysis of wave groups, in Proceedings of 25th International Conference on Offshore Mechanics and Arctic Engineering, (American Society of Mechanical Engineers, Hamburg, 2006), pp. $155-158$

3. R. Balaji, S. Sannasiraj, V. Sundar, Detection of wave groups from the motion behaviour of a discus buoy. J. Hydro-Environ. Res. 1(3), 195-205 (2008)

4. R. Balaji, S. Sannasiraj, V. Sundar, Identification of modal parameters of a floating system from impulse motion. Ocean Eng. 35, 1560-1564 (2008)

5. R. Balaji, S. Sannasiraj, V. Sundar, Identification of breaking events from the responses of a data buoy. J. Eng. Marit. Environ. 224, 127-139 (2010)

6. M.A.K. Elasyed, A novel technique in analyzing nonlinear wavewave interaction. Ocean Eng. 33, 168-180 (2006)

7. M.A.K. Elasyed, Wavelet bicoherence analysis of wind-wave interaction. Ocean Eng. 33, 458-470 (2006)

8. M.A.K. Elasyed, On the linear and nonlinear interaction between wind and wave. J. Coastal Res. 24(2), 519-526 (2008)

9. Z. Ge, P.C. Liu, Long term wave growth and its linear and nonlinear interactions with wind fluctuations. Ann. Geophys. 26, 747-758 (2008)

10. B. Mehak, R. Balaji, Historical wave characteristics along Mumbai coast during south-west monsoon. Curr. Sci. (2015) (Under review)

11. M. Farge, Wavelet transforms and their applications to turbulence. Annu. Rev. Fluid Mech. 1992(24), 395-457 (1992) 